\title{
Rasch Analysis of the Kentucky Inventory of Mindfulness Skills
}

\section{Oleg N. Medvedev ${ }^{a}$, Richard J. Siegert ${ }^{a}$, Paula Kersten ${ }^{b}$ and Christian U. Krägeloh ${ }^{a}$}

a Auckland University of Technology, New Zealand, Faculty of Health and Environmental Sciences

b University of Brighton, UK, School of Health Sciences

Address of corresponding author:

Oleg Medvedev

Department of Psychology

Faculty of Health \& Environmental Sciences

Auckland University of Technology - North Shore Campus

Private Bag 92006

Auckland 1142

New Zealand

Tel.: +64 99219999 ext 7661

Fax: +64 99219780

Email: oleg.medvedev@aut.ac.nz

Email address of other authors:

Professor Richard Siegert: richard.siegert@aut.ac.nz

Professor Paula Kersten: p.kersten@brighton.ac.uk

Professor Christian Krägeloh: chris.krageloh@aut.ac.nz

\section{Acknowledgements}

This study is a part of doctoral work of the first author funded by the Vice-Chancellor's

Scholarship of the Auckland University of Technology. 


\title{
Rasch Analysis of the Kentucky Inventory of Mindfulness Skills
}

1

\begin{abstract}
The current study reports Rasch analysis conducted to enhance the psychometric properties of the Kentucky Inventory of Mindfulness Skills (KIMS), the widely-used 39-item multidimensional measure of four mindfulness traits including: Observing, Describing, Act With Awareness, and Accept Non-Judgementally. While this instrument has generally robust psychometric properties, the ability of its subscales to precisely discriminate between trait levels and the functioning of individual items, have not been rigorously investigated. We subjected the responses of 287 participants to Rasch analysis and found that the Rasch model fits KIMS subscales after minor modifications that involved rescoring one item, removing misfitting items and combining locally dependent items into subtests. Precision of the KIMS can be improved substantially by using the proposed 34-item version of the instrument together with the ordinal-to-interval conversion tables presented here, without any need to modify the original response format. These findings can be useful for clinicians applying mindfulness-based interventions and researchers investigating neurophysiological and psychological correlates of trait mindfulness.
\end{abstract}

Keywords: Mindfulness, Measurement, Kentucky Inventory of Mindfulness Skills, Rasch analysis, Psychometrics 


\section{Introduction}

Mindfulness practice is a non-invasive method for the management of stress, emotional problems and for the improvement of psychological well-being (Baer, 2003; Brown \& Ryan, 2003). In the context of psychotherapy, mindfulness can be defined as "the non-judgmental observation of the on-going stream of internal and external stimuli as they arise” (Baer, 2003, p. 125). Considering the most cited definitions, mindfulness refers to paying attention to and being aware of internal and external experiences of the present moment associated with a non-judgmental attitude and may include friendliness, acceptance, kindness, curiosity and allowing (Segal, Williams, \& Teasdale, 2013). There has been a surge in the application of mindfulness training for a wide range of psychological and health conditions. Indeed, there is a rapidly growing evidence base for the therapeutic application of mindfulness techniques for alleviating symptoms and enhancing the coping abilities of people suffering from anxiety, stress, depression, emotional instability, substance abuse, post-traumatic stress disorder, borderline personality disorder, psychophysiological disorders, and suicidal/self-harm behavior (Chiesa \& Serretti, 2010; Hofmann, Sawyer, Witt, \& Oh, 2010; Zoogman, Goldberg, Hoyt, \& Miller, 2014). Also, mindfulness-based interventions (MBIs) were reported to enhance psychological well-being (Bennet \& Dorje, 2015; Josefsson, Lindwall, \& Broberg, 2014) and regulation of emotions (Chambers, Gullone, \& Allen, 2009; Lyvers, Makin, Tomas, Thorberg, \& Samios, 2014). Trait mindfulness was implicated as an important predictor in different models of psychological wellbeing (Brown \& Kasser, 2005; Pearson, Brown, Bravo, \& Witkiewitz, In Press) and hence its baseline levels should be controlled when evaluating outcomes of MBIs (Visted, Vøllestad, Nielsen, \& Nielsen, 2015). Consequently, there is a need for well-validated and precise mindfulness measures that accurately reflect psychological changes in people participating in MBIs. 
The Kentucky Inventory of Mindful Skills (KIMS) was developed as a multi-dimensional self-report measure of mindfulness-related skills introduced in the context of Dialectical Behavior Therapy (DBT) (Baer, Smith, \& Allen, 2004). DBT treatment unifies mindfulness of non-judgmental observation derived from Zen Buddhism with Western contemplative traditions (Hayes, Follette, \& Linehan, 2004). At the beginning of therapy, the goal is to develop individual skills of observing thoughts, emotions, and external stimuli by describing them. DBT emphasizes acting with awareness as a skill by cultivating it through a series of exercises that develop a routine of focusing attention on activities. Non-judgemental acceptance is also a primary skill that is recognized as part of the therapeutic process. To foster this skill, patients are encouraged to accept their reality and tolerate any unwanted feelings or thoughts without judgement (Linehan, 1993a, 1993b). It was proposed that mindfulness-related facets measured by the KIMS have utility in therapeutic contexts because they allows professionals to separate areas of skill development and, accordingly, assist individuals in strengthening specific skills (Baer et al., 2004).

It should be noted that unlike mindfulness-based stress reduction (Kabat-Zinn, 1982, 1990) and mindfulness-based cognitive therapy (Segal, Williams, \& Teasdale, 2002), which emphasize the central role of mindfulness in the therapeutic process, DBT includes mindfulness as a subcomponent among other treatment tools to increase sensory and perceptual awareness in normal, non-meditative circumstances (Hayes, Strosahl, \& Wilson, 1999; Linehan, 1993a). For example, the Describe subscale would not be consistent with the most cited mindfulness definitions used in psychology to design mindfulness measures (Bishop et al., 2006; Kabat-Zinn, 1994; Segal et al., 2013), which limits comparisons of the KIMS with other mindfulness measures. The exception is the Five Facets Mindfulness Questionnaire (FFMQ) (Baer, Smith, Hopkins, Krietemeyer, \& Toney, 2006), which has some structural similarities with the KIMS and was constructed from the combined items of five mindfulness scales including the KIMS (Baer et al., 2004), the Mindful Attention and Awareness Scale (MAAS) (Brown \& Ryan, 2003), the Freiburg Mindfulness Inventory (Buchheld, 
Grossman, \& Walach, 2001; Walach, Buchheld, Buttenmuller, Kleinknecht, \& Schmidt, 2006), the Southampton Mindfulness Questionnaire (Chadwick et al., 2008), and the Cognitive and Affective Mindfulness Scale (Feldman, Hayes, Kumar, Greeson, \& Laurenceau, 2007). However, the FFMQ has five subscales compared to the four of KIMS and includes items that are not specifically designed for the measurement of mindfulness skills utilized in a DBT context.

The KIMS includes 39 items divided into four subscales: Accept Non-Judgementally, Observe, Act With Awareness, and Describe (Baer et al., 2004). Accept Non-Judgementally is a subscale of the KIMS that measures the judging behavior present in individuals, such as self-criticism. Observe is a subscale that measures the degree of attention an individual pays to both external events and internal emotions, sensations, and cognitions. Act With Awareness assesses the individual's ability to be fully attentive to the present moment. Describe is a subscale that measures an individual's predisposition to describe or label their external and internal experiences. The items are presented in a 5-point Likert scale format, with responses ranging from 'Never or very rarely true' $=1$ to 'Very often or always true' = 5. Examples of typical items reflecting the four skills include: "I tell myself I shouldn't be feeling the way I'm feeling" (Non-Judgementally), "I notice when my mood changes" (Observe), "I tend to do several things at once" (Act With Awareness), and "I find words to describe my feelings” (Describe). Evidence indicates acceptable internal consistency for the total KIMS scale and all subscales, and good test-retest reliability for all subscales ( $r=.81$ to .86 ), with the exception of Observe $(\mathrm{r}=.65)$. In an exploratory factor analysis, $43 \%$ of variance in the data was explained by four factors, which was interpreted as support for the four-factor model of the KIMS (Baer et al., 2004). Confirmatory factor analysis supported the four-factor model but failed to confirm an overarching second-order mindfulness factor (Baer et al., 2004; Baum et al., 2010).

Convergent and divergent validity of the total KIMS and its subscales Non-Judgementally and Act With Awareness were supported by positive correlations with self-compassion, openness, and 
emotional intelligence and negative correlations with mindlessness, neuroticism, and dissociation (Baer et al., 2006). However, these relationships appear less consistent for the Observe and the Describe subscales (Baer et al., 2004; Christopher \& Gilbert, 2010; Frewen, Evans, Maraj, Dozois, \& Partridge, 2008). Describing internal and external experiences may have a therapeutic value although it does not feature in most psychological definitions of mindfulness (Baer, 2003; Kabat-Zinn, 1994; Segal et al., 2013). The main limitations of the KIMS include relatively low correlations (ranging from 0.09 to 0.34 ) between subscales (Baer et al., 2004) and concerns related to the content validity (e.g. the Describe subscale) (Park, Reilly-Spong, \& Gross, 2013). To date, no reports are available about investigations into the psychometric properties of the KIMS using modern item-response theory and in particular the Rasch model. The ultimate goal of a Rasch analysis is conversion from ordinal-level data to interval level, which increases measurement precision and permits parametric statistical analyses without violation of their assumptions (Brogden, 1977; Rasch, 1961). Generally, only few reported studies so far have subjected mindfulness measures to Rasch analysis (Goh, Marais, \& Ireland, 2015; Inchausti, Prieto, \& Delgado, 2014; Medvedev et al., 2015; Sauer, Ziegler, Danay, Ives, \& Kohls, 2013). While overall these studies provided useful diagnostic information of the FMI and English and Spanish versions of the MAAS at both individual item and scale levels, only one study has published the conversion tables that allow a reader to transform raw scores from the ordinal MAAS scale to an interval level data (Medvedev et al., 2015).

Rasch analysis can be beneficial to improve the precision of the instrument, given its distinct advantages over classical psychometric methods which have been well argued elsewhere (Rasch, 1960; Wilson, 2005; Wright \& Stone, 1979). Rasch analysis involves a unidimensional measurement model (Rasch, 1961) and in the case of the KIMS, Rasch analysis will be applied to each of the four KIMS subscales individually due to multidimensionality, low correlation between the subscales, and lack of support for an overarching mindfulness factor (Baer et al., 2004; Baum et al., 2010). 
Rasch analysis investigates several parameters of the model including local independence assumptions, potential item bias, unidimensionality, and stochastic ordering of items and response options in polytomous items (Tennant \& Conaghan, 2007). When fit to the Rasch model is achieved, these parameters satisfy the model requirements and participants can be ordered according to their ability or level on the latent trait (in this case one of the mindfulness skills). Similarly, items are ordered by difficulty or level of the specific mindfulness trait assessed by an item. In Rasch analysis, both the items and the participants are ordered on the same log-odds interval scale. Therefore, a participant-item threshold distribution can be presented graphically to illustrate how well the range of item difficulty covers or targets the abilities of the sample. The other benefit of Rasch analysis is identification of item bias or Differential Item Functioning (DIF). DIF is evident if participants with the same level on the latent trait, but from different groups (e.g. females and males), respond differently to an item.

The KIMS (Baer et al., 2004) is a widely-used multidimensional measure of four mindfulness traits with generally accepted psychometric properties. The main purpose of the KIMS was to be used in mindfulness-based treatment and studies, and it is critical to establish precision of its subscales. However, the ability of the KIMS subscales to precisely discriminate between trait levels and the functioning of individual items has not been investigated rigorously. Rasch analysis is a suitable method to investigate the performance of individual items to discriminate on their overarching trait, but to date Rasch analysis has not been used to study the psychometric properties of the KIMS subscales. The aim of this study is to apply Rasch analysis to investigate the psychometric properties of the KIMS and to explore strategies to improve precision and item functioning of its subscales.

\section{Method}

Participants 
This study analyzed data from a sample of 287 New Zealand university students $(78.7 \%$ females, 19.2\% males, 2.1\% missing gender). The mean age was 23.05 ( $\mathrm{SD}=7.64)$, with ages ranging from 18 to 59. Ethnicities included 51.3\% Caucasians, 8.7\% Māori, 7.7\% Pasifika, 19.7\% Asians, and 12.2\% of unspecified others. The sample size met recommended optimal sample size estimates for Rasch analysis (Linacre, 1994). To investigate DIF, two age categories were created: $18-20$ ( $\mathrm{n}=149)$ and 21-59 $(\mathrm{n}=127)$. There were $43(15 \%)$ individuals regularly engaging in mindfulness practice, as opposed to 240 not engaging in regular practice, and 4 individuals with data missing. Therefore, DIF was tested for the person factors including gender, ethnic group, age, and engagement in meditation and relaxation practices, where meditation practice refers to regularly performing formal meditation exercises and relaxation practices refers to regular exercises such as yoga or progressive muscle relaxation.

\section{Procedure}

Potential participants were approached in lectures and invited to complete the survey and to hand the survey back to the researchers or submit it to a locked collection box at their respective faculty. Students completed the questionnaire in class before the lecture or during a break. The authors' university ethics committee approved this study.

\section{Measures}

The Kentucky Inventory of Mindfulness Skills (KIMS) is a 39-item self-report questionnaire developed to capture the four mindfulness skills acquired in DBT treatment including Accept NonJudgementally, Observe, Act With Awareness and Describe (Baer et al., 2004). There are 16 negatively worded items measuring absence of mindfulness skills including items $3,4,8,11,14,16$, 18, 20, 22, 24, 27, 28, 31, 32, 35 and 36. These items were reversed coded prior to statistical analysis. 


\section{Data Analysis}

Prior to Rasch analysis, basic psychometric properties including reliability and factor structure of the KIMS were tested to be used for later comparisons with the Rasch results. Descriptive statistics, reliability analysis, and exploratory factor analysis were completed using IBM SPSS v.22, and Rasch analysis was completed using the software RUMM2030 (Andrich, Sheridan, \& Luo, 2009). Rasch analysis is a unidimensional measurement model that involves testing of unidimensionality along with other psychometric criteria. Therefore, unidimensionality of the KIMS subscales and their fit to the Rasch model were analyzed separately for each subscale of the KIMS including: Accept NonJudgementally, Observe, Describe, and Act With Awareness. First, the likelihood-ratio test was conducted on the initial analysis output for each subscale to confirm appropriateness of the unrestricted (Partial-Credit) version of the model. Rasch analyses followed ten main steps described elsewhere (Siegert, Tennant, \& Turner-Stokes, 2010):

1. Overall goodness of fit test of the data to the Rasch model.

2. Identifying and rescoring disordered thresholds.

3. Identifying and removing items not fitting to the Rasch model.

4. Re-test of overall and individual item fit to the Rasch model.

5. Examining Differential Item Functioning (DIF) for personal factors (e.g. gender).

6. Unidimensionality test of the four KIMS subscales.

7. Examining the residual correlation matrix for local dependency.

8. Inspection of the participant-item thresholds distribution plot.

9. Equating test between the final solution for a subscale (steps 1-8) and the original subscale.

10. Post-hoc comparisons between Rasch results and traditional psychometric criteria (e.g. reliability, factor analysis).

Rasch analysis starts with the overall evaluation of the model fit followed by identifying any items 
displaying disordered thresholds by examining the threshold map. A threshold is disordered when higher ability of an individual on a latent trait (e.g. Observe) is not consistently reflected in a gradual increase in ordinal response options for that specific item. Typically, ordering of thresholds is achieved by collapsing closest response categories. Overall goodness of fit to the model is usually retested after items with disordered thresholds have been rescored. After ordering of thresholds is completed, items showing the poorest fit to the model are removed one at a time and the overall model fit is again tested.

Rasch analysis follows this iterative process until achieving the following criteria: Both overall and individual item fit to the model are acceptable, and unidimensionality is clearly evident. Item-trait interaction indexed by an overall and individual item chi-square fit statistic should be not significant ( $>0.05$, Bonferroni adjusted). Also, overall person and item fit-residuals should have a mean close to 0.00 and a standard deviation close to 1.00 in the case of an excellent fit. Individual items are expected to have fit residuals in the range between -2.50 and +2.50 . All items should be locally independent, which is tested by examining the residual correlations between items. According to the conservative estimation (Marais \& Andrich, 2008), the residual correlations between items exceeding 0.20 above the mean of all residual correlations are indicative of local dependency. If residual correlations between items are above this level, instead of removing locally dependent items, these items are combined into a subtest (Wainer \& Kiely, 1987), and the overall model fit is once again examined. Also, the Rasch model requires no significant differences in DIF (Bonferroni adjusted) for age, gender, and other personal factors. If any of the examined items shows DIF for a specific personal factor, that item can be split into relevant categories, without the need to remove it (Wainer \& Kiely, 1987), followed by re-testing of the overall model fit. Dimensionality is examined by an independent-samples $t$ test comparing person-locations for two groups of items with the highest positive and the highest negative loadings on the first principal component after a latent trait (Rasch) factor is removed (Smith, 2002). Unidimensionality was tested for each subscale 
individually because of multidimensionality and weak relationships between subscales of the KIMS. For instance, if a subscale has 10 items then the items with the highest positive factor loadings above 0.2 will form one set (e.g. 3 items) and equal number of items with the highest negative loadings (e.g. 3 items) will form another set. Then the estimates of each individual on these two sets of items will be compared by paired-samples $t$ tests meaning that $287 t$ tests will be conducted for the current sample ( $n=287)$. Then the percentage of significant $t$ tests will be computed together with the + 95\% binominal confidence interval (CI). If the lower bound of the binominal CI computed for this percentage of significant $t$ tests is smaller than $5 \%$ (e.g. 4\%), then, based on statistical convention of alpha 0.05 , we do not have any reason to believe that there is a real difference between the estimates, and unidimensionality is therefore evident. Additionally, reliability of the subscales was assessed by the person separation index (PSI) in Rasch analysis, which measures the ability of a scale to discriminate between groups at different levels of the latent trait. PSI is numerically close to Cronbach's alpha and similarly estimates the error-free proportion of variance of person estimates distribution relative to the total variance including error. Unlike Cronbach's alpha, PSI calculation involves non-linear transformation of the raw scores and can be performed with random missing data, which is an advantage of Rasch analysis.

\section{Results}

Exploratory factor analysis (EFA) using the principal axis factoring extracted ten factors with eigenvalues above 1.00. However, the largest amount of variance in the data (44\%) was explained by just four factors and supported by the clear cut-off point on the scree-plot, which was consistent with the original report (Baer et al., 2004). For that reason, the number of extracted factors was fixed to four. Applying Varimax rotation with Kaiser normalization yielded the factor loadings presented in Table 1, with items generally following the factor structure of the original study (Baer et al., 2004). The internal consistency of the full 39-item scale was satisfactory with Cronbach's alpha of 0.82 . However, some individual item-to-total correlations for the full scale were low, ranging from -0.19 
to 0.50 (mean $r=0.29$ ), with two items falling below 0.10 (item 8, "I tend to evaluate whether my perceptions are right or wrong”, r=-0.19, and item 19, "When I do things, I get totally wrapped up in them and don't think about anything else”, $r=0.03$ ). The low correlations (0.10 to 0.30$)$ found between the subscales were consistent with the original validation report (Baer et al., 2004) and provide additional evidence for multidimensionality of the KIMS.

\section{<PLEASE INSERT TABLE 1 ABOUT HERE>}

Table 2 shows means and standard deviations for meditators and non-meditators together with Cronbach's alpha coefficients for each subscale of the KIMS. Cronbach's alpha for the subscales was in the acceptable range with an exception of the Act With Awareness subscale $(\alpha=0.65)$. According to expectations, significantly higher mean values were observed for meditators compared to non-meditators, with the exception of Accept Non-Judgmentally subscale as evidenced by subsequent $t$ tests (Observe: $t(276)=-4.49, p<.001$; Describe: $t(274)=-2.42, p=.016$; Act With Awareness: $t(274)=-2.26, p=.024)$.

\section{<PLEASE INSERT TABLE 2 ABOUT HERE>}

\section{Rasch Analysis for Accept Non-Judgementally Subscale}

The initial model fit statistics for the 9-item Accept Non-Judgementally subscale are presented in Table 3. PSI of 0.88 confirmed satisfactory reliability of the subscale, and none of the items displayed disordered thresholds. However, the overall model fit was poor $\left(\chi^{2}(36)=157.43, p<0.001\right)$, and item 8 displayed an extremely high fit residual of 8.08, well above the 2.50 cut-off point (Table 1). Table 1 shows the location or difficulty of each item on the Rasch scale in probability units or logits.

Deletion of item 8 resulted in a satisfactory overall model fit $\left(\chi^{2}(40)=45.43, \mathrm{p}>0.05\right)$ and acceptable reliability (PSI=0.89) of the subscale. Following the deletion of item 8, item 24 also exhibited an unacceptably high fit-residual of 3.64 and was therefore also removed before the analysis continued. 
The overall model fit improved after the deletion of item $24\left(\chi^{2}(35)=34.89, p=0.47\right)$, and no other misfitting items were identified. At this stage, the residual correlation matrix was examined, and local dependencies were found between items 4 and 12, and between items 16 and 32, as evidenced by residual correlations exceeding the 0.20 limit above the mean of all residual correlations. After combining two pairs of locally dependent items into two subtests, the overall good fit to the Rasch model was further improved $\left(\chi^{2}(25)=20.97, p=0.69\right.$, Table 3, 'Accept Non-Judgementally', Final Analysis). At this stage, all individual items had acceptable fit to the model, and no other locally dependent items could be identified.

\section{$<$ PLEASE INSERT TABLE 3 ABOUT HERE $>$}

To test unidimensionality, the set of person estimates from the items with the highest positive loadings on the first principal component were compared with the set of estimates from the items with the highest negative loadings by an independent-samples $t$ test. After calibrating $t$ tests between both sets of estimates to the same metric, $20 \mathrm{t}$ test comparisons out of 287 (6.97\%) were significant. A binominal test was used to calculate the precise amount of acceptable deviations for the current sample. Unidimensionality of the final solution was confirmed by the overlap of the $5 \%$ cutoff point on the lower bound surrounding $t$ test (Table 3, 'Accept Non-Judgementally', Final Analysis). No DIFs were found for person factors including gender, ethnic group, age, and engagement in meditation and relaxation practices.

The person-item threshold distribution plot for the Accept Non-Judgementally subscale (Final Analysis) is presented in Figure 1. The plot represents the relationship between distribution of item difficulty and person ability on the latent trait (e.g. Accept Non-Judgementally) converted to the same metric in logit units. Distribution of person thresholds is close to normal with some signs of 
ceiling and floor effects. However, over $90 \%$ of the sample was adequately covered by the items of the modified subscale.

\section{<PLEASE INSERT FIGURE 1 ABOUT HERE $>$}

Rasch Analysis for Observe Subscale

Initial analysis conducted for the 12-item Observe subscale yielded acceptable chi-square $\left(\chi^{2}(48)=63.02, \mathrm{p}=0.07\right)$ and reliability $(\mathrm{PSI}=0.82)$ values. However, $\mathrm{t}$ test comparisons between two sets of estimates with highest and lowest loadings on the first principal component after removing the latent trait component failed to confirm unidimensionality, with $8.71 \%$ of significant $\mathrm{t}$ tests and lower bound overlap above 5\% (Table 4). Also, item 29 displayed disordered thresholds and needed to be rescored before the analysis continued. After collapsing response options "Never or very rarely true” and "Rarely true”, and "Sometimes true” and "Often true”, thresholds of item 29 were precisely ordered. Figure 2 illustrates item response probability curve for item 29 before and after rescoring.

\section{<PLEASE INSERT FIGURE 2 ABOUT HERE $>$}

The overall model fit was slightly improved after rescoring item $29\left(\chi^{2}(48)=62.12, p=0.09\right)$, and all individual items showed a good fit to the Rasch model (Table 4, Observe). However, unidimensionality of the subscale was not confirmed. Unidimensionality of a scale can be compromised by locally dependent items, which can be identified by residual correlations between them. Therefore, the residual correlation matrix was examined, and local dependency was found between items 21 and 25, and items 30 and 37, which were then combined into two subtests. This minor modification produced the final solution for this subscale, with overall good fit to the model $\left(\chi^{2}(40)=40.50, \mathrm{p}=0.45\right)$ and acceptable reliability (PSI=0.80) (Table 4). A binominal test to test unidimensionality of the final solution indicated overlap on the lower bound surrounding t test with the $5 \%$ cutoff point, which confirmed unidimensionality (Table 4, 'Observe', Final Analysis). No 
DIF was noted for personal factors, such as gender, ethnic group, age, and meditation and relaxation practices.

Figure 1 shows the item-person threshold distribution for the final solution of the Observe subscale. Overall, person thresholds are distributed close to normal and well targeted by item threshholds, but there are some signs of a small ceiling effect. In this analysis, a good fit of the Observe subscale to the Rasch model was achieved with the minor modifications of rescoring one item and creating two subtests, without a need to remove any misfitting items.

\section{Rasch Analysis for Describe Subscale}

Initial analysis of the 8-item Describe subscale indicated acceptable reliability (PSI=0.85) but an overall lack of fit to the model $\left(\chi^{2}(32)=54.40, \mathrm{p}=0.008\right)$ and lack of evidence for unidimensionality (Table 4). At this stage no items displayed unacceptably high fit residuals. Therefore, the residual correlation matrix was examined, indicating local dependency between items 14, 18, and 22, which were then combined into a subtest before analysis continued. After creating the subtest, the chisquare had a lower but still significant value $\left(\chi^{2}(24)=39.12, \mathrm{p}=0.03\right)$. However, at the individual item level, item 6 displayed a high fit residual of 2.81, above the 2.50 cut-off point and was removed, which resulted in a good overall model fit $\left(\chi^{2}(20)=22.52, p=0.31\right)$ and continued acceptable reliability (PSI=0.78) (Table 4, Describe, Final). At the individual item level, all items showed acceptable fit to the model. Unidimensionality of the final solution was confirmed by the binominal test indicating overlap on the lower bound surrounding $t$ test with the $5 \%$ cutoff point. No significant DIFs were found for personal factors.

The person-item threshold distribution for the final analysis of the Describe subscale shows acceptable targeting of the person locations by the item thresholds (Figure 1). However, a slight ceiling effect was apparent indicating some limitation of the subscale in measuring higher personal 
abilities on Describe. Thus, satisfactory fit to the model was evident after few modifications that involved combining locally dependent items into a subtest and removal of one non-fitting item.

\section{Rasch Analysis for Act With Awareness Subscale}

Initial testing of the 10-item Act With Awareness subscale of the KIMS revealed an overall lack of fit to the model with a significant chi-square for overall person-trait interaction $\left(\chi^{2}(40)=67.39\right.$, $\mathrm{p}=0.004$ ) and reliability (PSI) of 0.66 (Table 4, Act With Awareness, Initial). The assumption of unidimensionality was violated as indicated by the binominal test not overlapping the $5 \%$ cutoff point on the lower bound. Consequently, individual item fit statistics were examined, indicating that item 35 had a significantly high fit residual $\left(\chi^{2}(4)=15.75, p<0.001\right)$. Item 35 was thus deleted, resulting in acceptable overall model fit, with a non-significant chi-square value $\left(\chi^{2}(36)=41.20\right.$, $\mathrm{p}=0.25$ ) but a slight reduction in reliability (PSI=0.61). Additionally, evidence for unidimensionality was lacking as the binominal test indicated no overlap with the $5 \%$ cutoff point on the lower bound. To test for local dependency, the residual correlation matrix was examined showing that item 19 had high residual correlations with items 7 and 38 that exceeded the 0.20 cut-off point above the mean of all residual correlations. Item 19 also had the lowest item-to-total correlation of 0.19 (Table 4) and so was removed resulting in the final solution with an acceptable chi-square value $\left(\chi^{2}(4)=38.40\right.$, $\mathrm{p}=0.202$ ) and evidence for unidimensionality (Table 4, Act With Awareness, Final). However, the reliability of the Act With Awareness subscale decreased slightly after these modifications (PSI=0.60). At this stage, all individual items were showing good fit to the model, and no DIF was identified for any personal factors.

The bottom panel of Figure 1 shows the item-person thresholds distribution for the final solution of the Act With Awareness subscale, with fairly good coverage of the range of individuals locations by the subscale items thresholds. Thus, the Act With Awareness subscale modified by removing two misfitting items satisfied all but reliability criteria for fitness to the Rasch model. 


\section{Ordinal-to-Interval Scale Conversion Table}

Table 4 includes conversion scores from an ordinal-to-interval level scale for all four subscales of the KIMS. For convenience, all the scores are adjusted to the scoring algorithm of each original subscale, and the total ordinal score is calculated by adding the scores of all the individual items included in a final version of a subscale after negatively worded items are reverse coded. Also, item 29 has to be rescored according to the algorithm at the bottom of Table 4, before calculating the total score for the Observe subscale. Therefore, ordinal scores are represented on the left-hand side and corresponding Rasch interval-level scores on the right-hand side (Table 4). The conversion table provided here allows users to increase precision of the KIMS subscales without the need to modify the original response format of the scale. These conversions can only be used when there are no missing data.

\section{<PLEASE INSERT TABLE 4 ABOUT HERE $>$}

\section{Discussion}

The KIMS (Baer et al., 2004) is a multidimensional measure of mindfulness widely used to assess four mindfulness-related skills: Observing, Describing, Act With Awareness, and Accept NonJudgementally. These mindfulness skills are linked to therapeutic outcomes of MBIs and especially in the context of DBT (Baer et al., 2004; Dimidjian \& Linehan, 2003). Our intention was not to assess the overall construct validity of the KIMS as such reports are already available (Baer et al., 2004; Park et al., 2013) but rather to perform investigation and fine-tuning for each individual subscale of the KIMS and test their structural validity using Rasch analysis. Thus, the aim of the current study was to use strategies of Rasch analysis to improve precision and item functioning of the KIMS subscales.

Given the fact that the subscales of the KIMS are commonly found only to be loosely related to each other (Baum et al., 2010), a finding confirmed in the present study, we focused our analyses on the 
subscales. Accuracy of any ordinal scale is limited, but can be improved up to an interval level using the Rasch model (Tennant \& Conaghan, 2007). The results of this analysis show that precision and item functioning of the KIMS subscales can be improved substantially using the Rasch model. Satisfactory fit to the Rasch model was achieved by a few modifications of the KIMS subscales that involved rescoring one item, deleting non-fitting items and combining locally dependent items into subtests (Table 4). Generally, the results support structural validity and reliability of the modified KIMS subscales to measure mindfulness skills in the sample population. However, initially low reliability of Act With Awareness subscale remained at the level of 0.60, indicating that the subscale is unable to distinguish between 2 strata with different ability levels (Fisher, 1992), which limits its applicability. The Rasch analysis confirms unidimensionality of all four modified KIMS subscales meaning that raw scores can be readily transformed into interval level scores using the same metric (Table 4) as long as there are no missing data. Clinicians and researchers may use the conversion algorithms of Table 4 to transform ordinal data into interval-level scores to investigate precise effects of MBIs on specific mindfulness traits.

The modification of the KIMS subscales involved rescoring of only one item (item 29) that displayed disordered thresholds in the Observe subscale, which indicates that the response options selected by the authors (Baer et al., 2004) were overall appropriate. Also, only five items needed to be removed as they did not fit the Rasch model. No items were removed from the Observe subscale, and only item 6 ("I can easily put my beliefs, opinions, and expectations into words”) was removed from the Describe subscale due to its poor fit. This item might not fit well to the model because it is not focused on describing experiences, which is the primary target of the Describe subscale. Also, focusing on describing beliefs, opinions, and expectations could be seen as moving away from the present moment and thus associated with less mindfulness. Two semantically close non-fitting items were deleted from the Accept Non-Judgementally subscale, namely item 8 ("I tend to evaluate 
whether my perceptions are right or wrong”) and item 24 (“I tend to make judgments about how worthwhile or worthless my experiences are”). Item 8 had the lowest item-to-total correlation for both the full scale ( $r=-0.19)$ and its subscale $(r=0.21)$, and it is the most difficult item where just few individuals scored high. Item 24 also seems to measure dichotomous judgement but is worded in a more adaptive way. These items might not work well with the current sample because they focus on extreme dichotomous judgement attitudes perhaps more common among people with borderline personality disorder (Linehan, 1993a, 1993b). Finally, two non-fitting items were removed from the Act With Awareness subscale: item 19 ("When I do things, I get totally wrapped up in them and don’t think about anything else”) and item 35 ("When I'm working on something, part of my mind is occupied with other topics, such as what I'll be doing later, or things I'd rather be doing”). Item 19 had very low item-to-total correlations for the full scale $(r=0.03)$ and its subscale $(r=0.16)$ and seems to measure one-pointed concentration rather than mindfulness (Brown, Ryan, \& Creswell, 2007; Olendzki, 2005). Item 35 seems to be a negatively worded counterpart of item 19 and it might also lead to bias due to its relatively complex wording. Future studies might examine how rewording of these items may improve psychometric properties of the KIMS subscales. However, until such investigation has been completed, it is recommended to use the proposed subscales versions together with the ordinal-to-interval scoring algorithm.

Removing non-fitting items resulted in the 34-item KIMS version that included the following modified subscales: the 7-item Accept Non-Judgementally, the 7-item Describe and the 8-item Act With Awareness. The original 12-item Observe subscale retained all its items after the modification. Combining locally dependent items into subtests was beneficial in achieving the satisfactory model fit for the subscales Observing, Describing, and Accept Non-Judgementally, without the need to discard more items. The psychometric properties of the modified KIMS subscales are, therefore, improved substantially with the exception of Act With Awareness subscale, which had initially low internal consistency that could not be improved by the current modifications. 
Unlike in the Rasch analysis of the MAAS (Medvedev et al., 2015), where uniform rescoring of all items was conducted to correct disordered thresholds, the KIMS items displayed no disordered thresholds, with an exception of item 29, which supports psychometric properties of the KIMS. Similarly to the MAAS, no more than two items were removed per subscale to achieve a satisfactory fit to the Rasch model, thus providing support for overall good structural validity of both scales. Also, four items (6, 8, 24 and 35) out of the five non-fitting items identified through Rasch analysis of the KIMS were included in the FFMQ constructed through factor analysis of the available mindfulness questionnaires (Baer et al., 2006), and it might be worthwhile to investigate functioning of those items also in the FFMQ using Rasch analysis. In addition, consistent with the earlier reports (Park et al., 2013), significantly higher mean scores were found for meditators compared to nonmeditators for all but Accept Non-Judgmentally subscale of KIMS, which supports construct validity of these subscales.

The following limitations should be noted. The study was conducted with a single sample of university students and should be replicated with more diverse samples including clinical and general populations. The analyses might also have been affected by degree of missing data as well as disproportional distribution of gender, age, and formal meditation experience in the sample. Although the sample reflects New Zealand's diversity of ethnic groups (Statistics New Zealand, 2013), no efforts were made to purposively sample underrepresented groups. Even though a satisfactory fit to the Rasch model was achieved for all subscales of the KIMS, the reliability of Act With Awareness subscale could not be improved, and item 29 required rescoring before computing a total score of the Observe subscale. However, if you have complete data, conversion from ordinal to an interval level scale can be conducted simply by adding responses on each modified subscale version and selecting a corresponding interval score in the right column (Table 4). The benefits of this conversion certainly outweigh inconvenience, and the authors can be contacted if assistance with data conversion is necessary. In addition, an Excel spreadsheet will be provided online to simplify 
data conversion.

Mindfulness skills have emerged as important contributors of therapeutic outcomes and their accurate assessment represents an on-going challenge. The current study reported Rasch analysis conducted to advance psychometric properties of the widely-used KIMS, a multidimensional measure of four mindfulness traits. We demonstrated that the KIMS subscales are structurally (or internally) valid after modifications that involved rescoring item 29, removing misfitting items 6, 8, 19, 24, and 35, as well as combining locally dependent items into subtests. Precision of the KIMS can be improved substantially by using the proposed 34-item version of the instrument together with the ordinal-to-interval conversion table presented here (Table 4), without any need to modify the original response format. These findings will be of interest for clinicians applying mindfulness-based interventions and researchers investigating neurophysiological and psychological correlates of trait mindfulness.

\section{Compliance with Ethical Standards}

We declare that we have no conflicts of interest in connection with this paper. The study was conducted in compliance with the guidelines of the authors’ university ethics committee.

\section{References}

Andrich, D., Sheridan, B., \& Luo, G. (2009). RUMM 2030. Perth: RUMM Laboratory.

Baer, R. (2003). Mindfulness training as a clinical intervention: a conceptual and empirical review. Clinical Psychology: Science and Practice, 10(2), 125-142.

Baer, R., Smith, G., Hopkins, J., Krietemeyer, J., \& Toney, L. (2006). Using self-report assessment methods to explore facets of mindfulness. Assessment, 13(1), 27-45.

Baer, R. A., Smith, G. T., \& Allen, K. B. (2004). Assessment of mindfulness by self-report: the Kentucky Inventory of Mindfulness Skills. Assessment, 11(3), 191-206. 
Baum, C., Kuyken, W., Bohus, M., Heidenreich, T., Michalak, J., \& Steil, R. (2010). The

Psychometric Properties of the Kentucky Inventory of the Mindfulness Skills in Clinical Populations. Assessment, 17(2), 220-229.

Bennett, K., \& Dorjee, D. (2015). The impact of a mindfulness-based stress reduction course (MBSR) on well-being and academic attainment of sixth-form students. Mindfulness. doi:10.1007/s12671-015-0430-7

Bishop, S. R., Lau, M., Shapiro, S., Carlson, L., Anderson, N. D., Carmody, J., ... ,\& Devins, G. (2006). Mindfulness: a proposed operational definition. Clinical Psychology: Science and Practice, 11(3), 230-241.

Brogden, H. E. (1977). The Rasch model, the law of comparative judgment and additive conjoint measurement. Psychometrika, 42(4), 631-634.

Brown, K. W., \& Kasser, T. (2005). Are psychological and ecological well-being compatible? The role of values, mindfulness, and lifestyle. Social Indicators Research, 74(2), 349-368.

Brown, K. W., \& Ryan, R. M. (2003). The benefits of being present: mindfulness and its role in psychological well-being. . Journal of Personality and Social Psychology, 84(4), 822-848. Brown, K. W., Ryan, R. M., \& Creswell, J. D. (2007). Mindfulness: theoretical foundations and evidence for its salutary effects. Psychological Inquiry, 18(4), 211-237.

Buchheld, N., Grossman, P., \& Walach, H. (2001). Measuring mindfulness in insight meditation (vipassana) and meditation-based psychotherapy: the development of the Freiburg Mindfulness Inventory (PMI). Journal for Meditation and Meditation Research, 1, 11-34.

Chadwick, P., Hember, M., Symes, J., Peters, E., Kuipers, E., \& Dagnan, D. (2008). Responding mindfully to unpleasant thoughts and images: reliability and validity of the Southampton Mindfulness Questionnaire (SMQ). The British Journal of Clinical Psychology, 47(4), 451-455. 
Chambers, R., Gullone, E., \& Allen, N. B. (2009). Mindfulness emotion regulation: an integrative review. Clinical Psychology Review, 29(6), 560-572.

Chiesa, A., \& Serretti, A. (2010). A systematic review of neurobiological and clinical features of mindfulness meditations. Psychological Medicine, 40(8), 1239-1252.

Christopher, M. S., \& Gilbert, B. D. (2010). Incremental validity of components of mindfulness in the prediction of satisfaction with life and depression. Current Psychology, 29(1), 10-23.

Dimidjian, S., \& Linehan, M. M. (2003). Defining an agenda for future research on the clinical application of mindfulness practice. Clinical Psychology: Science and Practice, 10(2), 166171.

Feldman, G., Hayes, A., Kumar, S., Greeson, J., \& Laurenceau, J.-P. (2007). Mindfulness and emotion regulation: the development and initial validation of the Cognitive and Affective Mindfulness Scale-Revised (CAMS-R). Journal of Psychopathology \& Behavioral Assessment, 29(3), 177-190.

Fisher, W. (1992). Reliability statistics. Rasch Measurement Transactions, 6(3), 238.

Frewen, P. A., Evans, E. M., Maraj, N., Dozois, D. J. A., \& Partridge, K. (2008). Letting go: mindfulness and negative automatic thinking. Cognitive Therapy \& Research, 32(6), 758774.

Goh, H. E., Marais, I., \& Ireland, M. J. (2015). A Rasch model analysis of the Mindful Attention Awareness Scale. Assesment. doi:DOI: 10.1177/1073191115607043

Hayes, S. C., Follette, V. M., \& Linehan, M. M. (Eds.). (2004). Mindfulness and acceptance: expanding cognitive-behavioral tradition. New York: The Guilford Press.

Hayes, S. C., Strosahl, K., \& Wilson, K. G. (1999). Acceptance and commitment therapy: an experiential approach to behavior change. New York: Guilford Press. 
Hofmann, S. G., Sawyer, A. T., Witt, A. A., \& Oh, D. (2010). The effect of mindfulness-based therapy on anxiety and depression: a meta-analytic review. Journal of Consulting and Clinical Psychology, 78(2), 169-183.

Inchausti, F., Prieto, G., \& Delgado, A. R. (2014). Rasch analysis of the Spanish version of the Mindful Attention Awareness Scale (MAAS) in a clinical sample. Revista de Psiquiatría y Salud Mental, 7(1), 32-41.

Josefsson, T., Lindwall, M., \& Broberg, A. G. (2014). The effects of a short-term mindfulness based intervention on self-reported mindfulness, decentering, executive attention, psychological health, and coping style: examining unique mindfulness effects and mediators. Mindfulness, 5(1), 18-35.

Kabat-Zinn, J. (1982). An outpatient program in behavioural medicine for chronic pain patients based on the practice of mindfulness meditation: theoretical considerations and preliminary results. General Hospital Psychiatry, 4(1), 33-47.

Kabat-Zinn, J. (1990). Full catastrophe living: using the wisdom of your body and mind to face stress, pain and illness. New York, USA: Delacorte.

Kabat-Zinn, J. (1994). Wherever you go, there you are: mindfulness meditation in everyday life. New York, USA: Hyperion.

Linacre, J. M. (1994). Sample size and item calibration stability. Rasch Measurement Transactions, 7, 328.

Linehan, M. M. (1993a). Cognitive-behavioural treatment of borderline personality disorder. New York, USA: Guilford Press.

Linehan, M. M. (1993b). Skills training manual for treating bordeline personality disorder. New York, USA: Guilford Press.

Lyvers, M., Makin, C., Toms, E., Thorberg, F. A., \& Samios, C. (2014). Trait mindfulness in relation to emotional self-regulation and executive function. Mindfulness, 5(6), 619-625. 
Marais, I., \& Andrich, D. (2008). Effects of varying magnitude and patterns of response dependence in the unidimensional Rasch model. Journal of Applied Measurement, 9(2), 105-124.

Medvedev, O. N., Siegert, R. J., Feng, X. J., Billington, D. R., Jang, J. Y., \& Krägeloh, C. U. (2015). Measuring trait mindfulness: how to improve the precision of the Mindful Attention Awareness Scale using a Rasch model. Mindfulness. doi:10.1007/s12671-015-0454-z

Olendzki, A. (Ed.). (2005). The roots of mindfulness. New York, USA: Guilford.

Park, T., Reilly-Spong, M., \& Gross, C. R. (2013). Mindfulness: a systematic review of instruments to measure an emergent patient-reported outcome (PRO). Quality of Life Research, 22(10), 2639-2659.

Pearson, M. R., Brown, D. B., Bravo, A. J., \& Witkiewitz, K. (2015). Staying in the moment and finding purpose: the associations of trait mindfulness, decentering, and purpose in life with depressive symptoms, anxiety symptoms, and alcohol-related problems. Mindfulness, 6(3), 645-653.

Rasch, G. (1960). Probabilistic models for some intelligence and attainment test. Copenhagen: Danish Institute for Educational Research.

Rasch, G. (1961). On general laws and the meaning of measurement in psychology.University of California Press. Symposium conducted at the meeting of the Proceedings of the Fourth Berkeley Symposium on Mathematical Statistics and Probability., Berkeley, California.

Sauer, S., Ziegler, M., Danay, E., Ives, J., \& Kohls, N. (2013). Specific objectivity of mindfulness - a Rasch analysis of the Freiburg Mindfulness Inventory. Mindfulness, 4(1), 45-54.

Segal, Z. V., Williams, J. M. G., \& Teasdale, J. D. (2002). Mindfulness based cognitive therapy for depression. New York, USA: Guilford Press.

Segal, Z. V., Williams, J. M. G., \& Teasdale, J. D. (2013). Mindfulness-Based Cognitive Therapy for depression (2nd ed.). New York: Guilford Press. 
Siegert, R. J., Tennant, A., \& Turner-Stokes, L. (2010). Rasch analysis of the Beck Depression Inventory-II in a neurological rehabilitation sample. Disability and Rehabilitation, 32(1), 8-17.

Statistics New Zealand. (2013). Census ethnic group profiles. Wellington: New Zealand Government.

Tennant, A., \& Conaghan, P. G. (2007). The Rasch measurement model in rheumatology: what is it and why use it? When should it be applied, and what should one look for in a Rasch paper? Arthritis \& Rheumatism, 57(8), 1358-1362.

Visted, E., Vøllestad, J., Nielsen, M. B., \& Nielsen, G. H. (2015). The impact of group-based mindfulness training on self-reported mindfulness: a systematic review and meta-analysis. Mindfulness, 6(3), 501-522.

Wainer, H., \& Kiely, G. (1987). Item clusters and computerized adaptive testing: a case for testlets. Journal of Educational Measurement, 24(3), 185-201.

Walach, H., Buchheld, N., Buttenmüller, V., Kleinknecht, N., \& Schmidt, S. (2006). Measuring mindfulness - the Freiburg Mindfulness Inventory (FMI). Personality and Individual Differences, 40(8), 1543-1555.

Wilson, M. (2005). Constructing measures. Mahwah, NJ: LEA.

Wright, B. D., \& Stone, M. H. (1979). Best test design. Chicago, USA: MESA Press.

Zoogman, S., Goldberg, S. B., Hoyt, W. T., \& Miller, L. (2015). Mindfulness interventions with youth: a meta-analysis. Mindfulness, 6(2), 290-302. 

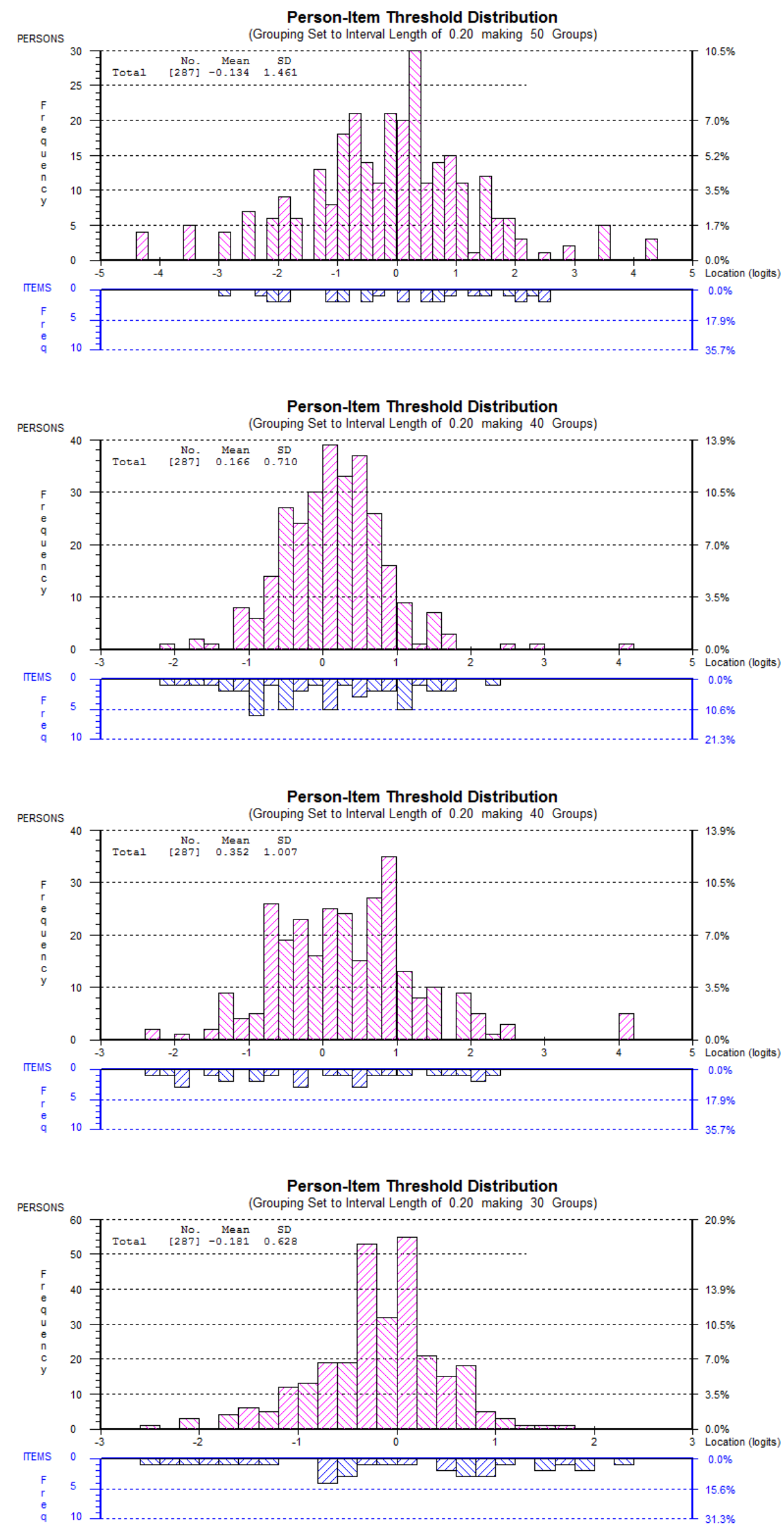

Figure 1. Person-item threshold distribution for modified KIMS subscales from top to bottom including Accept, Observe, Describe and Act $(n=287)$. 

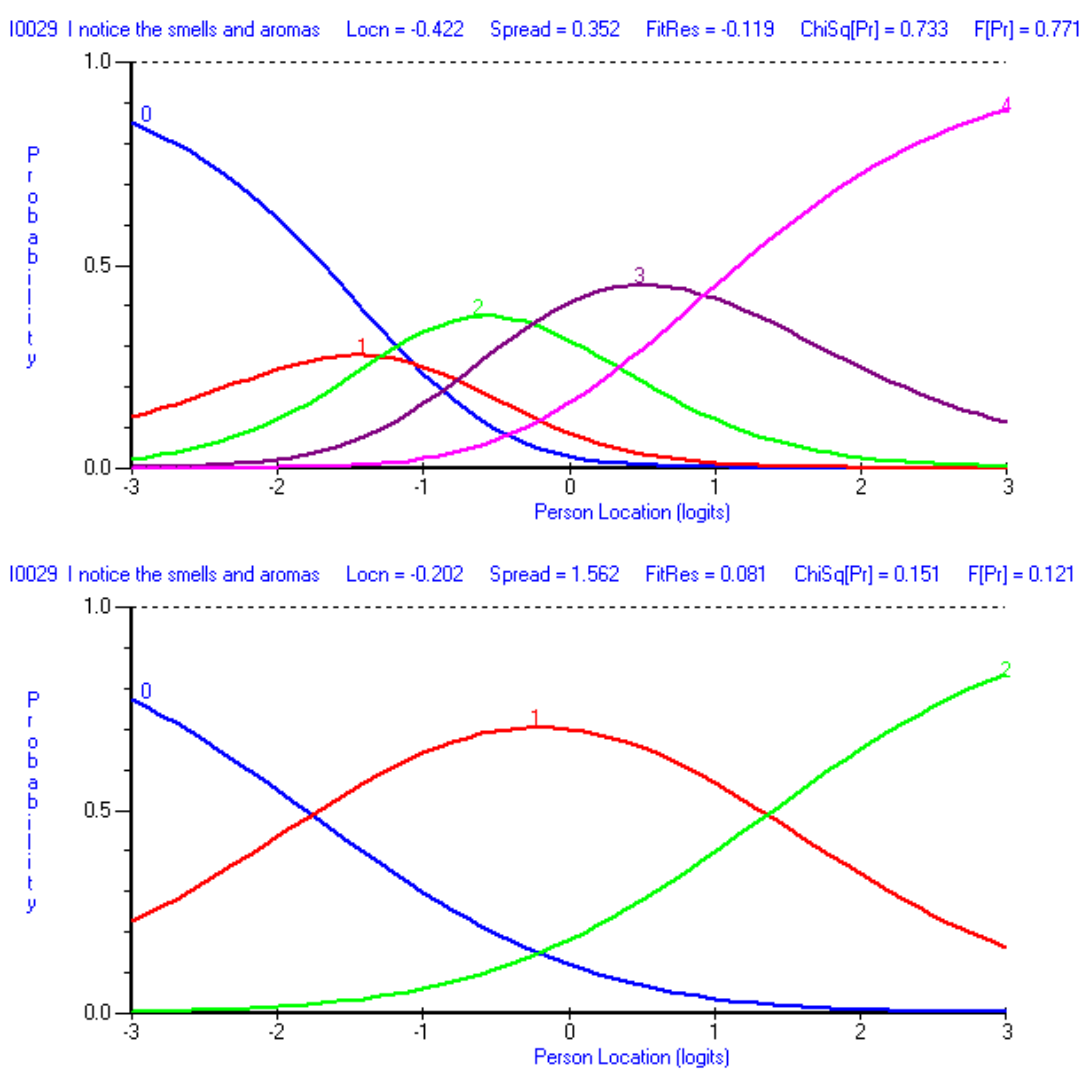

Figure 2. Item category probability curves illustrating disordered thresholds for KIMS item 29 before rescoring (top panel) and orderly thresholds after rescoring (bottom panel). 
Table 1. Initial item location, item fit residual, corrected item-to total correlation and factor loadings for Accept Non-Judgmentally (Accept), Observe, Describe and Act With Awareness (Act) subscale items of KIMS.

\begin{tabular}{|c|c|c|c|c|c|}
\hline $\mathbf{N}$ & Subscale/ Item & $\begin{array}{c}\text { Item } \\
\text { Location }\end{array}$ & $\begin{array}{l}\text { Item Fit } \\
\text { Residual }\end{array}$ & $\begin{array}{l}\text { Item- } \\
\text { Total }\end{array}$ & $\begin{array}{l}\text { Factor } \\
\text { Loading }\end{array}$ \\
\hline & Accept & & & & \\
\hline 4 & I criticize myself for having irrational or inappropriate emotions. ${ }^{\mathrm{R}}$ & 0.29 & -1.21 & 0.68 & 0.77 \\
\hline 8 & I tend to evaluate whether my perceptions are right or wrong. ${ }^{\mathrm{R}}$ & 0.46 & 8.08 & 0.21 & 0.27 \\
\hline 12 & I tell myself that I shouldn’t be feeling the way I'm feeling. ${ }^{\mathrm{R}}$ & 0.09 & -0.50 & 0.67 & 0.76 \\
\hline 16 & I believe some of my thoughts are abnormal or bad and I shouldn't think that way. ${ }^{\mathrm{R}}$ & -0.20 & -0.86 & 0.68 & 0.76 \\
\hline 20 & I make judgments about whether my thoughts are good or bad. ${ }^{\mathrm{R}}$ & 0.14 & -1.27 & 0.70 & 0.77 \\
\hline 24 & I tend to make judgments about how worthwhile or worthless my experiences are. ${ }^{\mathrm{R}}$ & 0.02 & 2.16 & 0.56 & 0.62 \\
\hline 28 & I tell myself that I shouldn't be thinking the way I'm thinking. ${ }^{\mathrm{R}}$ & -0.17 & -2.37 & 0.74 & 0.83 \\
\hline 32 & I think some of my emotions are bad or inappropriate and I shouldn't feel them. ${ }^{\mathrm{R}}$ & -0.42 & -2.47 & 0.73 & 0.82 \\
\hline \multirow[t]{2}{*}{36} & I disapprove of myself when I have irrational ideas. ${ }^{\mathrm{R}}$ & -0.21 & -0.45 & 0.68 & 0.74 \\
\hline & Observe & & & & \\
\hline 1 & I notice changes in my body, such as whether my breathing slows down or speeds up. & -0.22 & 1.10 & 0.42 & 0.49 \\
\hline 5 & I pay attention to whether my muscles are tense or relaxed. & 0.62 & 0.07 & 0.49 & 0.53 \\
\hline 9 & When I’m walking, I deliberately notice the sensations of my body moving. & 0.72 & 0.60 & 0.47 & 0.60 \\
\hline 13 & When I take a shower or a bath, I stay alert to the sensations of water on my body. & 0.17 & 1.23 & 0.45 & 0.56 \\
\hline 17 & I notice how foods and drinks affect my thoughts, bodily sensations, and emotions. & 0.19 & 0.87 & 0.43 & 0.50 \\
\hline 21 & I pay attention to sensations, such as the wind in my hair or sun on my face. & 0.11 & -1.37 & 0.58 & 0.71 \\
\hline 25 & I pay attention to sounds, such as clocks ticking, birds chirping, or cars passing. & 0.07 & 1.06 & 0.45 & 0.61 \\
\hline 29 & I notice the smells and aromas of things. & -0.42 & -0.12 & 0.49 & 0.63 \\
\hline 30 & I intentionally stay aware of my feelings. & -0.10 & -0.63 & 0.54 & 0.57 \\
\hline 33 & $\begin{array}{l}\text { I notice visual elements in art or nature, such as colors, shapes, textures, or patterns of light and } \\
\text { shadow. }\end{array}$ & -0.22 & 1.53 & 0.43 & 0.54 \\
\hline 37 & I pay attention to how my emotions affect my thoughts and behavior. & -0.43 & 0.12 & 0.48 & 0.49 \\
\hline 39 & Describe & & & & \\
\hline 2 & I'm good at finding the words to describe my feelings. & -0.04 & -1.33 & 0.65 & 0.76 \\
\hline 6 & I can easily put my beliefs, opinions, and expectations into words. & -0.41 & -1.34 & 0.68 & 0.73 \\
\hline 10 & I'm good at thinking of words to express my perceptions, such as how things taste, smell or sound & 0.18 & 1.36 & 0.56 & 0.55 \\
\hline 14 & It's hard for me to find the words to describe what I'm thinking. ${ }^{\mathrm{R}}$ & -0.01 & -2.24 & 0.69 & 0.78 \\
\hline 18 & I have trouble thinking of the right words to express how I feel about things. ${ }^{\mathrm{R}}$ & -0.02 & -2.16 & 0.72 & 0.79 \\
\hline 22 & $\begin{array}{l}\text { When I have a sensation in my body, it's difficult for me to describe it because I can't find the } \\
\text { right words. }\end{array}$ & -0.42 & 2.25 & 0.44 & 0.59 \\
\hline 26 & Even when I’m feeling terribly upset, I can find a way to put it into words. & 0.37 & 3.64 & 0.51 & 0.60 \\
\hline \multirow[t]{2}{*}{34} & My natural tendency is to put my experiences into words. & 0.36 & 3.10 & 0.48 & 0.56 \\
\hline & Act & & & & \\
\hline 3 & When I do things, my mind wanders off and I'm easily distracted. ${ }^{\mathrm{R}}$ & 0.22 & -0.77 & 0.43 & 0.54 \\
\hline 7 & When I'm doing something, I'm only focused on what I'm doing, nothing else. & -0.12 & -0.15 & 0.40 & 0.55 \\
\hline 11 & I drive on "automatic pilot” without paying attention to what I'm doing. ${ }^{\mathrm{R}}$ & -0.48 & 1.81 & 0.24 & 0.34 \\
\hline 15 & When I'm reading, I focus all my attention on what I'm reading. & -0.60 & 1.23 & 0.28 & 0.47 \\
\hline 19 & When I do things, I get totally wrapped up in them and don't think about anything else. & -0.20 & 1.56 & 0.16 & 0.44 \\
\hline 23 & $\begin{array}{l}\text { I don't pay attention to what I'm doing because I'm daydreaming, worrying, or otherwise } \\
\text { distracted. }\end{array}$ & -0.12 & -0.27 & 0.39 & 0.37 \\
\hline 27 & $\begin{array}{l}\text { When I'm doing chores, such as cleaning or laundry, I tend to daydream or think of other } \\
\text { things }^{\mathrm{R}}\end{array}$ & 0.59 & 0.93 & 0.24 & 0.42 \\
\hline 31 & I tend to do several things at once rather than focusing on one thing at a time. ${ }^{\mathrm{R}}$ & 0.21 & 0.42 & 0.31 & 0.49 \\
\hline 35 & $\begin{array}{l}\text { When I'm working on something, part of my mind is occupied with other topics, such as what } \\
\text { I'll be doing later, or things I'd rather be doing. }\end{array}$ & 0.52 & -1.64 & 0.49 & 0.62 \\
\hline 38 & I get completely absorbed in what I'm doing, so that all my attention is focused on it. & -0.02 & 0.70 & 0.26 & 0.47 \\
\hline
\end{tabular}

Note: ${ }^{\mathbf{R}}$ reverse-scored item. 
Table 2. Descriptive statistics for the Accept Non-Judgmentally (Accept), Observe, Describe and Act With Awareness (Act) subscales of KIMS including means and standard deviations (SD) for meditators and non-meditators, and Cronbach's alpha coefficients.

\begin{tabular}{lllllll}
\hline \multirow{2}{*}{ Subscale } & \multicolumn{2}{c}{ Meditators $(\mathrm{n}=42)$} & & \multicolumn{2}{c}{ Non-Meditators $(\mathrm{n}=221)$} & \multirow{2}{*}{ Cronbach's alpha } \\
\cline { 2 - 3 } & Mean & SD & & Mean & SD & \\
\cline { 2 - 3 } Accept & 25.40 & 7.74 & & 25.70 & 7.37 & 0.88 \\
Observe & 43.19 & 6.80 & & 37.76 & 7.31 & 0.82 \\
Describe & 26.90 & 4.91 & & 26.44 & 5.77 & 0.85 \\
Act & 29.29 & 5.60 & & 27.42 & 4.63 & 0.65 \\
\hline
\end{tabular}

Table 3. Summary of fit statistics for the initial and the final Rasch analyses of the four KIMS subscales Accept Non-Judgmentally (Accept), Observe, Describe and Act With Awareness (Act).

\begin{tabular}{|c|c|c|c|c|c|c|c|c|}
\hline \multirow[b]{2}{*}{ Analyses } & \multicolumn{2}{|c|}{$\begin{array}{l}\text { Item fit } \\
\text { residual } \\
\end{array}$} & \multicolumn{2}{|c|}{$\begin{array}{c}\text { Person fit } \\
\text { residual }\end{array}$} & \multicolumn{2}{|c|}{ Goodness of fit } & \multirow[t]{2}{*}{ PSI } & \multirow{2}{*}{$\begin{array}{c}\text { Independent } t \text {-test } \\
\% \mathrm{LB}^{\mathrm{a}}\end{array}$} \\
\hline & \multicolumn{2}{|c|}{ Value / SD } & \multicolumn{2}{|c|}{ Value / SD } & $\chi^{2}$ (df) & $p$ & & \\
\hline & \multicolumn{8}{|c|}{ Accept } \\
\hline Initial & 0.12 & 3.27 & -0.44 & 1.54 & $157.43(36)$ & $<0.001$ & 0.88 & 6.20 \\
\hline Final & 0.31 & 1.21 & -0.53 & 1.38 & $20.97(25)$ & 0.690 & 0.85 & 4.40 \\
\hline \multicolumn{9}{|l|}{ Observe } \\
\hline Initial & 0.52 & 0.93 & -0.29 & 1.40 & $63.02(48)$ & 0.070 & 0.82 & 7.20 \\
\hline Final & 0.55 & 0.62 & -0.28 & 1.30 & $40.50(40)$ & 0.450 & 0.80 & 4.80 \\
\hline \multicolumn{9}{|l|}{ Describe } \\
\hline Initial & 0.41 & 2.44 & -0.50 & 1.60 & $54.37(32)$ & 0.008 & 0.85 & 7.60 \\
\hline Final & 0.62 & 1.62 & -0.50 & 1.36 & $22.52(20)$ & 0.310 & 0.78 & 3.40 \\
\hline \multicolumn{9}{|l|}{ Act } \\
\hline Initial & 0.38 & 1.09 & -0.38 & 1.44 & $67.39(40)$ & 0.004 & 0.67 & 8.30 \\
\hline Final & 0.45 & 0.85 & -0.38 & 1.36 & $38.40(32)$ & 0.202 & 0.60 & 4.40 \\
\hline
\end{tabular}

Note: ${ }^{\mathrm{a}} \mathrm{LB}=$ lower bound of the $95-\%$ confidence interval. 
Table 4. Converting from ordinal to interval level scores for the subscales Accept Non-Judgmentally (Accept), Observe, Describe and Act With Awareness (Act) of the 34-item version of the KIMS.

\begin{tabular}{|c|c|c|c|c|c|c|c|c|c|}
\hline \multicolumn{2}{|c|}{ Accept Scores } & \multicolumn{4}{|c|}{ Observe Scores } & \multicolumn{2}{|c|}{ Describe Scores } & \multicolumn{2}{|c|}{ Act Scores } \\
\hline Ordinal & Interval & Ordinal & Interval & Ordinal & Interval & Ordinal & Interval & Ordinal & Interval \\
\hline 7 & 7.00 & 10 & 10.00 & 43 & 37.67 & 7 & 7.00 & 8 & 8.00 \\
\hline 8 & 9.72 & 11 & 14.49 & 44 & 38.22 & 8 & 9.84 & 9 & 11.19 \\
\hline 9 & 11.65 & 12 & 17.46 & 45 & 38.80 & 9 & 11.72 & 10 & 13.43 \\
\hline 10 & 13.02 & 13 & 19.43 & 46 & 39.40 & 10 & 12.96 & 11 & 15.01 \\
\hline 11 & 14.12 & 14 & 20.92 & 47 & 40.04 & 11 & 13.90 & 12 & 16.25 \\
\hline 12 & 15.06 & 15 & 22.11 & 48 & 40.74 & 12 & 14.70 & 13 & 17.29 \\
\hline 13 & 15.90 & 16 & 23.12 & 49 & 41.48 & 13 & 15.41 & 14 & 18.21 \\
\hline 14 & 16.66 & 17 & 24.00 & 50 & 42.31 & 14 & 16.07 & 15 & 19.02 \\
\hline 15 & 17.38 & 18 & 24.79 & 51 & 43.25 & 15 & 16.72 & 16 & 19.77 \\
\hline 16 & 18.06 & 19 & 25.50 & 52 & 44.32 & 16 & 17.36 & 17 & 20.46 \\
\hline 17 & 18.71 & 20 & 26.17 & 53 & 45.59 & 17 & 17.99 & 18 & 21.11 \\
\hline 18 & 19.34 & 21 & 26.78 & 54 & 47.17 & 18 & 18.61 & 19 & 21.73 \\
\hline 19 & 19.96 & 22 & 27.37 & 55 & 49.24 & 19 & 19.24 & 20 & 22.32 \\
\hline 20 & 20.57 & 23 & 27.92 & 56 & 52.36 & 20 & 19.86 & 21 & 22.89 \\
\hline 21 & 21.18 & 24 & 28.45 & 57 & 57.00 & 21 & 20.48 & 22 & 23.45 \\
\hline 22 & 21.79 & 25 & 28.97 & & & 22 & 21.10 & 23 & 23.99 \\
\hline 23 & 22.41 & 26 & 29.47 & & & 23 & 21.72 & 24 & 24.53 \\
\hline 24 & 23.03 & 27 & 29.97 & & & 24 & 22.34 & 25 & 25.06 \\
\hline 25 & 23.66 & 28 & 30.44 & & & 25 & 22.96 & 26 & 25.60 \\
\hline 26 & 24.30 & 29 & 30.92 & & & 26 & 23.58 & 27 & 26.13 \\
\hline 27 & 24.96 & 30 & 31.40 & & & 27 & 24.21 & 28 & 26.68 \\
\hline 28 & 25.66 & 31 & 31.87 & & & 28 & 24.85 & 29 & 27.24 \\
\hline 29 & 26.39 & 32 & 32.33 & & & 29 & 25.53 & 30 & 27.81 \\
\hline 30 & 27.19 & 33 & 32.80 & & & 30 & 26.29 & 31 & 28.41 \\
\hline 31 & 28.08 & 34 & 33.27 & & & 31 & 27.17 & 32 & 29.05 \\
\hline 32 & 29.13 & 35 & 33.73 & & & 32 & 28.26 & 33 & 29.73 \\
\hline 33 & 30.44 & 36 & 34.20 & & & 33 & 29.71 & 34 & 30.47 \\
\hline 34 & 32.32 & 37 & 34.68 & & & 34 & 31.85 & 35 & 31.30 \\
\hline \multirow[t]{5}{*}{35} & 35.00 & 38 & 35.16 & & & 35 & 35.00 & 36 & 32.25 \\
\hline & & 39 & 35.64 & & & & & 37 & 33.39 \\
\hline & & 40 & 36.13 & & & & & 38 & 34.84 \\
\hline & & 41 & 36.63 & & & & & 39 & 36.95 \\
\hline & & 42 & 37.14 & & & & & 40 & 40.00 \\
\hline
\end{tabular}

Note: Item 29 from the Observe subscale needs to be rescored before calculating the ordinal scores for this subscale as follows: $1=1$, $2=1,3=2,4=2,5=3$. Negatively worded items $3,4,8,11,14,16,18,20,22,24,27,28,31,32,35$ and 36 have to be reversed coded prior calculating the total score. For the Accept subscale, add items 4, 12, 16, 20, 28, 32, and 36. For the Observe subscale, add items $1,5,9,13,17,21,25,29,30,33,37$, and 39. For the Describe subscale, add items 2, 6, 10, 14, 18, 22, 26, and 34. For the Act subscale, add items $3,7,11,15,19,23,27,31$, and 38. For each subscale sum score, find the equivalent interval-level score in the above conversion table. This table cannot be used for respondents with missing data. 